\title{
SANS Investigation of PS-PB Block Copolymer Micelles in a Short Chain PB Homopolymer Matrix
}

\author{
Kerstin Gohr, ${ }^{\dagger}$ Wolfgang Schartl, ${ }^{, \dagger}$ Lutz Willner, ${ }^{\ddagger}$ and Wim Pyckhout-Hintzen‡ \\ Institut für Physikalische Chemie, Universität Mainz, Welderweg 11, 55099 Mainz, Germany, and \\ Institut für Festkörperforschung, Forschungszentrum J ülich, 52425 J ülich, Germany \\ Received J une 6, 2002
}

\begin{abstract}
A micelle forming polystyrene (PS)-polybutadiene (PB) block copolymer was mixed with a short (relative to the PB block of the copolymer) PB homopolymer to investigate the structural properties of the micelles by small-angle neutron scattering. Samples with varying block copolymer concentration were measured at $90^{\circ} \mathrm{C}$ in both core and shell contrast. The obtained intensities were analyzed using a core/shell model with a constant core density and a variable density profile for the corona. A reasonable analysis of the data was only possible by assuming that in the measured concentration range the unimer concentration is not constant but increases with increasing polymer concentration, while the aggregation number of the micelles stays constant. The data analysis further revealed that the corona of micelles shows almost constant density which stays in contrast to micelles of block copolymers with comparable block length ratio in low molecular solvents. These could be interpreted in terms of the starlike model of Halperin, where the outer shell has a fast decaying density profile.
\end{abstract}

\section{Introduction}

Block copolymer micelles embedded in a homopolymer matrix provide an interesting model system for soft colloidal particles. Whereas the dynamics of this system has been investigated in some detail, ${ }^{1-4}$ the particle structure itself, especially the density profile of the micellar corona, which directly should reflect the particle interaction potential, has not yet been studied. Importantly, the particle interactions and the corona dimensions in copolymer micelle/homopolymer blends can be adjusted via the homopolymer matrix. The "solvent quality" of the homopolymer depends on its molecular weight. Shorter chains show a better miscibility with the corona block of the copolymer. This effect is of purely entropic origin. There are no enthalpic contributions to the free energy of mixing because the polymer segments of corona and homopolymer are chemically identical. Theoretical calculations predict that there is an upper limit for homopolymer chains to penetrate into the micellar corona, which is the molecular weight of the corona block..$^{5-7}$ The shorter the matrix polymer chains, the better they mix with the micellar corona and, correspondingly, the stronger the swelling of the particle size. Previous studies have shown that this swelling decreases with increasing micelle concentration. ${ }^{4}$ To understand why swollen micelles shrink in effective size with increasing interactions, it is important to investigate the density profile of the copolymer corona in detail. ${ }^{8}$

Different models have been developed to describe the corona structure of micelles. ${ }^{9,10}$ All of them show that the block length ratio plays a very important role. In the case of short corona chains compared to the core block (crew cut micelles) the micelle can be regarded more or less as a planar brush because of the small curvature of the core surface compared to the corona thickness. Here, the segment density in the corona is

† Universität Mainz.

₹ Institut für Festkörperforschung.

* Corresponding author: e-mail schaertl@mail.uni-mainz.de. constant $(\rho(r)=$ const). On the other hand, if the corona block is much longer than the core block, the micelle is expected to show the behavior of a star molecule where the segment density in the corona is decaying with increasing distance from the core. Daoud and Cotton developed a model for star molecules to describe this decrease in corona density. ${ }^{11}$ They divide the corona chains into so-called blobs of size $\xi$ where the polymer shows single-chain behavior and has a constant density. The blob size increases with increasing distance $r$ to the core, which is equivalent to a decrease in density. This model leads to a radial density distribution of $\rho(r) \propto$ $r^{-4 / 3}$.

In between the two extremes there has to be a continuous transition. This could be shown experimentally by SANS experiments on PEP-PEO micelles of various compositions in water. ${ }^{12}$ The different density profiles could be modeled by a variable density profile of the following power law form:

$$
\rho(r) \propto r^{-x} /\left(1+\exp \left[\left(r-R_{M}\right) / \sigma\right]\right)
$$

By variation of the exponent $x$, the steepness of the decay can be adjusted. For $x=4 / 3$ eq 1 resembles the density profile of the Daoud and Cotton model. In the case of $x=0$ the other extreme of constant density is obtained. The Fermi function in the denominator has to be introduced to guarantee a cutoff of the density profile in the range of the micellar radius $\mathrm{R}_{M}$. Ther efore, this function considers the fact that the size of the micelle is limited by the chain dimensions. The parameter $\sigma$ modulates the smoothness of the cutoff.

In this study, we investigated blends with different copolymer content (0.5-7 wt \%) of an asymmetric micelle forming polystyrene-polybutadiene (PS-PB) block copolymer in a short PB homopolymer which is expected to strongly swell the micelle corona ("wet brush" regime). By SANS experiments in core and shell contrast, the structure of these blends has been analyzed, especially focusing on the effective particle size and the corona density profile. 
Table 1. Characteristics of the Block Copolymer and Homopolymers

\begin{tabular}{lll}
\hline \multicolumn{1}{c}{ polymer } & \multicolumn{1}{c}{$\mathrm{M}_{\mathrm{n}}^{\mathrm{a} / 10^{3}}$} & \multicolumn{1}{c}{$\mathrm{M}_{\mathrm{w}} / \mathrm{M}_{\mathrm{n}}{ }^{\mathrm{a}}$} \\
\hline dPS-hPB & $15^{\mathrm{b}} / 67\left(70^{\mathrm{c}}\right)$ & $<1.02^{\mathrm{b}} /<1.02$ \\
hPB8K & 7.9 & $<1.02$ \\
0.96d-0.04hPB8K & 8.8 & $<1.02$
\end{tabular}

a By SEC in THF. ${ }^{b}$ dPS block. ${ }^{c}$ By membrane osmometry in toluene.

\section{Experimental Section}

Polymers. The polymers used in this study were prepared by anionic polymerization under high vacuum in sealed glass reactors provided with break-seals for the addition of reagents. The techniques applied and the protocols for purification of initiator, monomers, and solvent were similar to those specified in the literature.13,14

The block copolymer dPS-hPB was synthesized by sequential addition of styrene- $d_{8}\left(98.5 \%\right.$ D) and butadiene- $h_{6}$. sec-Butyllithium served as initiator and benzene as solvent. Before the addition of the second monomer a small quantity of the living PS block was removed from the reactor for separate characterization. The polybutadiene homopolymers hPB8K for core contrast and 0.96d0.04hPB8K for shell contrast were polymerized with tert-butyllithium as initiator and benzene as solvent. The matrix polymer for shell contrast, $0.96 \mathrm{~d} 0.04 \mathrm{hPB} 8 \mathrm{~K}$, was made from an isotopic mixture consisting of $96 \%$ butadiene $\mathrm{d}_{6}(98 \% \mathrm{D})$ and $4 \%$ butadiene- $\mathrm{h}_{6}$ monomer in order to match the scattering length density of $\rho=6.4$ $\times 10^{10} \mathrm{~cm}^{-2}$ for dPS. An initiator-to-monomer ratio was adjusted such that the resulting molecular volume equals that of the fully protonated matrix polymer. The living polymers were terminated by the addition of a small amount of degassed methanol. The final products were obtained by precipitation in methanol followed by drying in a vacuum oven until constant weight was achieved.

The polymers were characterized by size exclusion chromatography (SEC) with a Waters 150C instrument. In addition to the internal refractive index detector a Waters 486 UV detector was connected in series. A set of four styragel columns covering a porosity range of $100-10^{4} \AA$ was chosen. Distilled tetrahydrofuran (THF) was the elution solvent at a flux of 1 $\mathrm{mL} / \mathrm{min}$. The molecular weight and the polydispersity of the materials were determined relative to polystyrene standards. Transformation to PB molecular weights was performed by means of the PS and PB Mark-Houwink-Sekurada relations $^{15,16} \mathrm{M}_{\mathrm{PB}}=0.581 \mathrm{M}_{\mathrm{PS}}{ }^{0.997}$. The higher molecular weight due to deuteration was considered during the calculation.

A comparison of the RI and UV chromatograms of the block copolymer indicates homogeneity of the composition over the elution peak. No sign of dPS homopolymer was found in the chromatograms corroborating that notermination has occurred during the addition of the second monomer. The numberaverage molecular weight $\left(M_{n}\right)$ of the block copolymer was additionally determined by membrane osmometry with a Knauer instrument in toluene at $37^{\circ} \mathrm{C}$. The molecular weight was extracted from a $(\Pi / \mathrm{c})^{1 / 2}$ vs c plot. The molecular characteristics of the polymers are summarized in Table 1.

SANS Measurements. The SANS studies were performed on the KWS1 instrument at the research reactor FRJ 2 at the F orschungszentrum J ülich $\mathrm{GmbH}$. The SANS samples were prepared by dissolving respective amounts of block copolymer and homopolymer in benzene followed by subsequent freezedrying under high vacuum until the solvent was completely removed. The blends were then transferred into quartz glass cuvettes with either $1 \mathrm{~mm}$ (core contrast) or $2 \mathrm{~mm}$ (shell contrast) path length. Transmission values larger than $45 \%$ were obtained.

The measurements were carried out at $90^{\circ} \mathrm{C}(=$ temperature regime of previous diffusion measurements of micellar systems by forced Rayleigh scattering, ${ }^{3,4}$ samples have been annealed prior to measurement for several hours) under vacuum to reduce background scattering using either three (shell contrast) or two (core contrast) spectrometer setups. F or shell contrast sample-to-detector distances of $L=2,8$, and 20 $\mathrm{m}$ were chosen. For 8 and $20 \mathrm{~m}$ the collimation was positioned at the same distance. For measurements at $2 \mathrm{~m}$ a collimation length of $8 \mathrm{~m}$ was taken in order to avoid detector dead time effects due to high neutron intensities and to increase the resolution. The neutron wavelength was $7 \AA$ with a spread of $\Delta \lambda / \lambda=20 \%$. Under these conditions a wave vector range of 3 $\times 10^{-3} \AA^{-1} \leq q \leq 0.15 \AA^{-1}$ was obtained with $q=(4 \pi / \lambda)$ sin $\Theta / 2$, where $\Theta$ denotes the scattering angle. In core contrast the measurements were mainly performed at 2 and $8 \mathrm{~m}$ sample-to-detector distance. The latter combination yields a q range of $8 \times 10^{-3} \AA^{-1} \leq q \leq 0.15 \AA^{-1}$, which is sufficient to cover all the important details of the form factors due to the smaller dimensions of the dPS core. F or incoherent background correction pure matrix polymers were measured separately in cuvettes with identical path lengths.

The raw data were corrected two-dimensionally for electronic background and empty cell scattering. Detector sensitivity corrections and transformation to absol ute scattering cross sections were made with a secondary Lupolene standard according to

$$
\frac{d \Sigma}{d \Omega}(q)=\left(\frac{d \Sigma}{d \Omega}\right)_{L} \frac{d_{L} T_{L} L_{S}{ }^{2}}{d_{S} T_{S} L_{L}{ }^{2} I_{L}}\left[\left(I_{S}-I_{C d}\right)-T_{S}\left(I_{E C}-I_{C d}\right)\right]
$$

where subscripts $\mathrm{L}$ and $\mathrm{S}$ stand for Lupolene and sample, respectively. $d$ and $T$ refer to cell thickness and transmission. $L_{s}$ and $L_{L}$ are detector distances at which sample and $L$ upolene were measured to give $\mathrm{I}_{\mathrm{S}}$ and $\mathrm{I}_{\mathrm{L}}$. Both are normalized to the same monitor counts to avoid a timely fluctuating flux. Since Lupolene is a flat, incoherent scatterer, I $\mathrm{L}$ was replaced by its average over the detector. The value of $(\mathrm{d} \Sigma / \mathrm{d} \Omega)_{\llcorner}=1.78155$ $\mathrm{cm}^{-1}$ for the Lupolene standard had been determined vs vanadium at $7 \AA$. The raw data were also corrected twodimensi onally for intensities of electronic background $I_{c d}$ and empty cell I IE. After proper correction, the data were radially averaged. Finally, the measured incoherent scattering of the matrix polymers and the theoretically calculated incoherent contribution from the protonated portion of the block copolymer were subtracted.

\section{Results and Discussion}

Critical Micelle Concentration in Copolymer/ Homopolymer Blends. The experimental scattering cross sections $\mathrm{d} \Sigma / \mathrm{d} \Omega(\mathrm{q})$ for the blends at various copolymer concentrations are shown in Figure 1. For easier comparison, the actual measured intensities have been normalized by the volume fractions of the labeled polymers, which is $\phi$ Ps for core contrast (Figure la) and $\phi_{\mathrm{hPB}}$ (Figure 1b) for shell contrast as given in Table 2.

In this concentration regime where the interparticle scattering (structure factor) is not yet pronounced and the measurements are dominated by intraparticle scattering (form factor), these normalized intensities should be identical for all concentrations. As Figure 1 shows, this is only the case in the high-q region. Within the accuracy of the experiment the curves for the different concentrations, especially in shell contrast, superimpose very well for $q>5 \times 10^{-2} 1 / \AA$. At high $q$ the scattering origi nates from the localized structure of the copolymer chains themselves: therefore, all copolymer chains present in the blend, i.e., isolated single copolymer chains and copolymer chains constituting a spherical micellar aggregate, contribute to the signal. Consequently, normalization to the volume fraction of the labeled block as given by the homopolymer/block copolymer composition of the blends leads to a good agreement of the normalized intensities measured at different block copolymer concentrations in the high-q regime. This, however, is different at small scattering 
a)

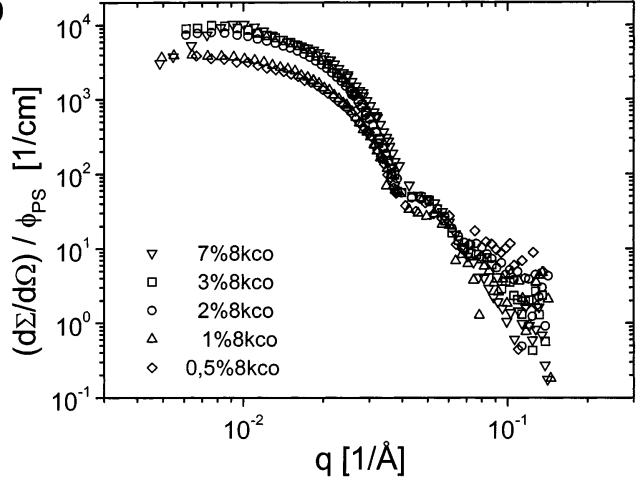

b)

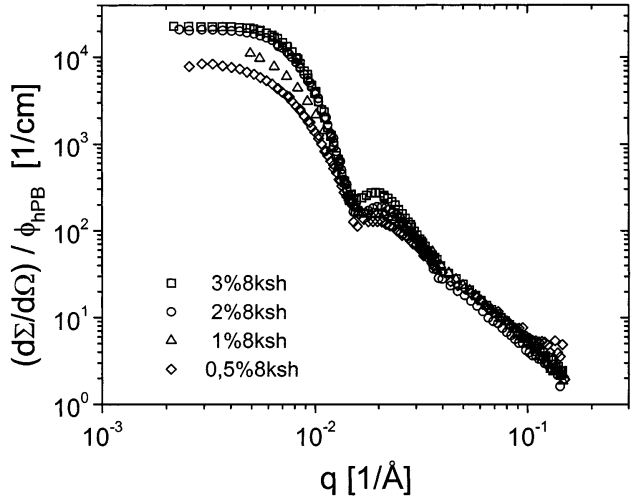

Figure 1. Scattering cross sections $\mathrm{d} \Sigma / \mathrm{d} \Omega(\mathrm{q})$ for the block copolymer/homopolymer blends at different concentrations in (a) core contrast and (b) shell contrast. The measured intensities have been normalized by the volume fraction of scattering polymer (a) $\phi_{\mathrm{PS}}$ and (b) $\phi_{\mathrm{hPB}}$.

Table 2. Composition of the Block Copolymer/ Homopolymer Blends

\begin{tabular}{ccc}
\hline core contrast & CSB $[w t ~ \%]$ & $\phi_{\mathrm{PS}}$ \\
\hline $0.5 \%$ 8K co & 0.55 & 0.0010 \\
$1 \%$ 8K co & 1.0 & 0.002 \\
$2 \% 8 \mathrm{~K}$ co & 2.2 & 0.004 \\
$3 \%$ 8K co & 3.0 & 0.005 \\
$7 \%$ 8K co & 6.6 & 0.012 \\
\hline shell contrast & CSB [wt \%] & $\phi_{\mathrm{hPB}}$ \\
\hline $0.5 \%$ 8K sh & 0.49 & 0.0043 \\
$1 \%$ 8K sh & 1.0 & 0.009 \\
$2 \%$ 8K sh & 2.0 & 0.017 \\
$3 \%$ 8K sh & 3.0 & 0.026
\end{tabular}

a In addition to the block copolymer content $\mathrm{CSB}_{\mathrm{SB}}$ in wt \% the volume fraction of the scattering polymer is given, which is $\phi \mathrm{PS}$ in the case of the core contrast and $\phi_{\mathrm{hPB}}$ for the shell contrast.

vectors q. Here, the normalized intensity decreases with decreasing block copolymer content. At small q, the larger structure of the micellar aggregates themselves is observed in the scattering signal. Therefore, the difference in the normalized scattering intensities in this q regime most probably is caused by assuming a wrong concentration of micelles: that is, the actual micelle concentration especially at very low copolymer concentration seems to be much lower than the total block copolymer concentration used for normalization of the scattering intensities.

A first analysis of the data for the 3 and 1 wt \% block copolymer blends was performed by fitting the data with a spherical core/shell model. Model and fitting procedures were analogous to those used for the structural analysis of PEP-PEO micelles. ${ }^{12}$ The aggregation number $n_{\text {agg }}$ (number of copolymer chains per micelle) was extracted from the scattering cross section $\mathrm{d} \Sigma / \mathrm{d} \Omega(\mathrm{q} \rightarrow 0)$

$$
\mathrm{d} \Sigma / \mathrm{d} \Omega(\mathrm{q} \rightarrow 0) \propto \Delta \rho^{2} \phi \mathrm{n}_{\mathrm{agg}}
$$

with $\Delta \rho^{2}=\left(\rho_{\mathrm{d}-\mathrm{PS}}-\rho_{\mathrm{h}-\mathrm{PB}}\right)^{2}=3.576 \times 10^{21} \mathrm{~cm}^{-4}$ as the contrast factor between d-PS and h-PB calculated by $\left(\Sigma_{\mathrm{d}-\mathrm{S}} / v_{\mathrm{d}-\mathrm{S}}-\Sigma_{\mathrm{h}-\mathrm{B}} / v_{\mathrm{h}-\mathrm{B}}\right)^{2}$, where $\Sigma_{\mathrm{d}-\mathrm{S}}$ and $\Sigma_{\mathrm{h}-\mathrm{B}}$ denote the sum of coherent scattering lengths of individual atoms in the repeat unit of the polymers with $v_{\mathrm{d}-\mathrm{S}}$ and $v_{\mathrm{h}-\mathrm{B}}$ as their respective volumes.

The core radius $R_{c}$ was a fit parameter obtained from the sphere form factor in core contrast. The results show a strong decrease from $n_{\text {agg }}=112$ for 3 wt $\%$ to $n_{\text {agg }}=$ 36 for 1 wt \% block copolymer concentration, while the core radius with $R_{c}=120 \AA$ and $R_{c}=110 \AA$, respectively, remained almost constant. Here, it should be stressed again that for this analysis $\phi$ has been determined from the total copolymer concentration, which obviously leads to results with nonphysical meaning.

In addition to the variation of the aggregation number at constant core radius with concentration there is al so a very large discrepancy to the aggregation number $\mathrm{n}_{\text {agg,core }}$ expected from the micelle core radius under the assumption that the core consists completely of polystyrene (strong segregation):

$$
\mathrm{n}_{\text {agg, core }}=\frac{\mathrm{m}_{\mathrm{PS}}}{\mathrm{M}_{\mathrm{S}}} \mathrm{N}_{\mathrm{L}}=\frac{4 \pi}{3} \frac{\rho_{\mathrm{PS}}}{\mathrm{M}_{\mathrm{S}}} \mathrm{R}_{\mathrm{c}}^{3}
$$

$\rho_{\mathrm{PS}}$ is the bulk density of PS. Equation 4 yields an aggregation number of $250-300$ chains per micelle at a core radius of $100-120 \AA$. These values are much larger than the numbers obtained from the preliminary analysis.

In principle, our findings could be explained by a weak segregation between the PS core and the PB corona of the micelles; that is, the micellar core of radius 100$120 \AA$ consists to a large extent of PB. This would decrease the intensity due to the change in contrast and would also decrease the aggregation number in relation to the core radius. But, considering the obtained aggregation numbers of 112 and 36, these would correspond to a butadiene content of about $70-90 \%$ in the core. For such a weak segregation the formation of micelles, which clearly are identified in our scattering signals, would be very improbable.

Therefore, another explanation seems more appropriate: in particular, at very low block copolymer concentration only part of the copolymer chains form micellar aggregates, leading to the wrong assumption of $\phi$ during the data normalization and analysis according to eq 3 , as al ready mentioned above. If we consider the amount of aggregated chains as determined from the apparent aggregation numbers, $\mathrm{n}_{\text {agg, }}$ in comparison to the value expected from the micelle core radius assuming strong segregation, $\mathrm{n}_{\text {agg,core, }}$ in the $3 \mathrm{wt} \%$ blend only about $50 \%$ of the block copolymer chains are built into micelles. For a copolymer concentration of $1 \mathrm{wt} \%$ the amount is even lower (15\%). This leads to the conclusion that the block copolymer concentration in our blends is very close to the minimum concentration where micelles are formed (critical micelle concentration).

As the amount of free block copolymer chains could not be neglected, single chain scattering has been incorporated in the fitting procedure used for a more detail ed analysis of our data. The total scattering cross section $\mathrm{d} \Sigma / \mathrm{d} \Omega(\mathrm{q})$ therefore was divided into two parts: 


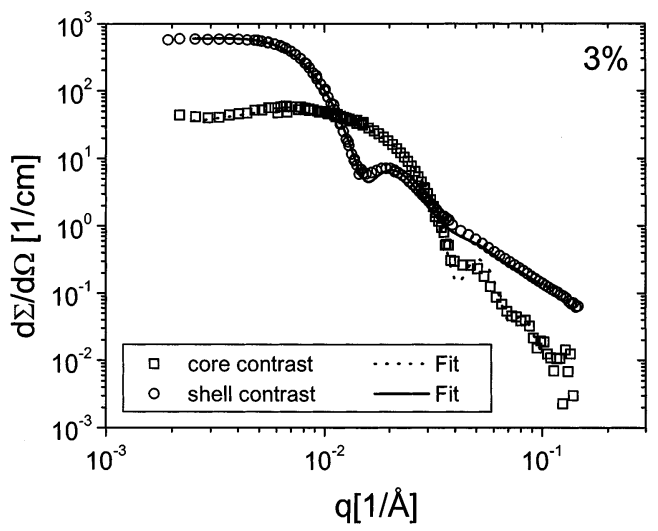

Figure 2. Example of fit result for the $3 \%$ blend. The figure shows the measured data and the corresponding fits for both core and shell contrast.

the micellar scattering $(\mathrm{d} \Sigma / \mathrm{d} \Omega)_{\text {mic }}$ and the unimer scattering $(\mathrm{d} \Sigma / \mathrm{d} \Omega)_{\text {uni, }}$, where the different contributions are weighted by the volume fractions of the scattering species, respectively.

$$
\begin{array}{r}
\left(\frac{\mathrm{d} \Sigma}{\mathrm{d} \Omega}\right)=\frac{\phi_{\mathrm{uni}}}{\phi_{\mathrm{SB}}}\left(\frac{\mathrm{d} \Sigma}{\mathrm{d} \Omega}\right)_{\mathrm{uni}}+\left(\frac{\phi_{\mathrm{mic}}}{\phi_{\mathrm{SB}}}\right)\left(\frac{\mathrm{d} \Sigma}{\mathrm{d} \Omega}\right)_{\mathrm{mic}}=\frac{\phi_{\mathrm{uni}}}{\phi_{\mathrm{SB}}}\left(\frac{\mathrm{d} \Sigma}{\mathrm{d} \Omega}\right)_{\mathrm{uni}}+ \\
\left(1-\frac{\phi_{\mathrm{uni}}}{\phi_{\mathrm{SB}}}\right)\left(\frac{\mathrm{d} \Sigma}{\mathrm{d} \Omega}\right)_{\text {mic }}
\end{array}
$$

with

$$
\phi_{\mathrm{SB}}=\phi_{\mathrm{mic}}+\phi_{\mathrm{uni}}
$$

Here, the volume fraction of free chains $\phi_{\text {uni }}$ is a fit parameter. In shell contrast, the unimer scattering $(\mathrm{d} \Sigma /$ $\mathrm{d} \Omega)_{\text {uni }}$ was considered with a Beaucage form factor ${ }^{17,18}$ for a polymer chain with the length of the PB corona block. In core contrast, the scattering contribution from single isolated block copolymer chains was neglected, because the PS part of an isolated block copolymer should form a tiny collapsed coil which nonsignificantly contributes to the overall intensity. The scattering from the micelles in core contrast is model ed by a solid sphere form factor ${ }^{19}\left(R_{c}\right)$. Additionally, the incorporation of a hard-sphere structure factor (parameters: hard-sphere volume fraction $V_{H S}$, effective hard-sphere radius $R_{H S}$ ) was necessary because a structure factor peak caused by interparticle interactions was visible in particular at higher concentrations in core contrast (see Figure la). Scattering curves in shell contrast are fitted with a form factor for a hollow sphere with the variable density profile described by eq 1 (parameters: $\mathrm{R}_{\mathrm{M}}, \mathrm{x}, \sigma$ ), but no additional structure factor. To ensure the consistency of the fits for core and shell contrast at a given copolymer concentration, both signals were fitted with identical parameters $R_{c}$ and $\phi_{\text {uni. }}$. The aggregation number $n_{\text {agg }}$ is calculated from the core radius according to eq 4. As an example, the resulting fits are shown for the 3\% blends in Figure 2.

The graph shows good agreement of the data with the corresponding fits. It was possible to fit all the data sets with only one core radius of $R_{c}=113 \AA$, which corresponds to an aggregation number of $n_{\text {agg }}=265$. The results for the other fit parameters are summarized in Table 3. Estimated error bars are less than $5 \%$ of the given values.

As can be seen from the values given for $\phi_{\text {uni }}$ in Table 3 , a considerable amount of the added polymer does not

\begin{tabular}{|c|c|c|c|c|c|c|}
\hline \multirow[b]{2}{*}{$\phi_{\mathrm{SB}}$} & \multirow[b]{2}{*}{$\phi_{\text {uni }}$} & \multirow{2}{*}{$\begin{array}{c}\text { shell form } \\
\text { factor } \\
R_{M}[\AA]\end{array}$} & \multicolumn{2}{|c|}{$\begin{array}{l}\text { density profile } \\
\text { (see eq 1) }\end{array}$} & \multicolumn{2}{|c|}{ structure factor } \\
\hline & & & $x$ & $\sigma$ & $\mathrm{V}_{\mathrm{HS}}$ & $\mathrm{R}_{\mathrm{HS}}[\AA]$ \\
\hline 0.005 & 0.0043 & 250 & 0.72 & 0.18 & & \\
\hline 0.01 & 0.0077 & & 0.77 & 0.23 & 0.025 & 337 \\
\hline & 0.0108 & 240 & 0.53 & 0.25 & 0.093 & 378 \\
\hline 0.03 & 0.0170 & 231 & 0 & 0.20 & 0.073 & 416 \\
\hline 0.07 & 0.0295 & & & & 0.256 & 301 \\
\hline
\end{tabular}

Table 3. Fit Results for All Block Copolymer Concentrations ${ }^{\mathrm{a}}$

a The core radius for all blends is $R_{c}=113 \AA$ ( $n_{\text {agg }}=265$ ). For the highest concentration only the core contrast was measured. The blend of the lowest block copolymer concentration showed no structure factor.

a)

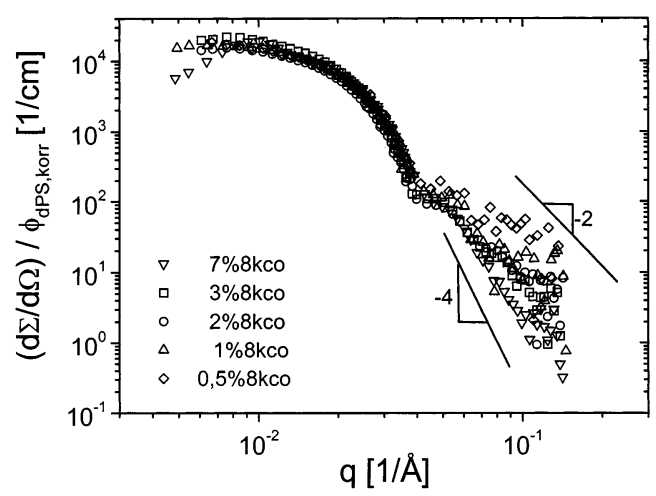

b)

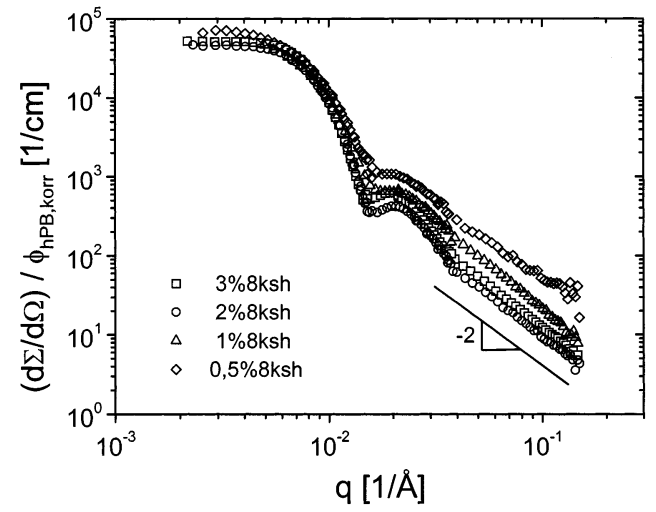

Figure 3. Normalized scattering cross sections after the correction of concentration for the unimer content: (a) core contrast, (b) shell contrast (compare Figure 1).

aggregate into micelles. Only at block copolymer concentrations above $2 \mathrm{wt} \%$, more than $50 \%$ of the chains are forming aggregates. If the data are normalized to the corrected volume fractions $\phi_{\mathrm{PS}, \text { corr }}$ and $\phi_{\mathrm{hPB}, \text { corr }}$, considering only the amount of block copolymer found within micel lar aggregates, the graphs shown in Figure 3 are obtained.

The deviations for low q observed in Figure 1 are not any longer visible in these plots, neither in core (Figure 3a) nor in shell contrast (Figure 3b). However, in the high-q regime the scattering intensity varies with concentration: in shell contrast, at lower copolymer concentration, where a major part of the copolymer is not aggregated into micelles, a higher normalized intensity is found because here the scattering intensity originates from both the PB part of the unimers (not considered in the normalization!) and the PB part in the micellar corona. In core contrast, the differences in scattering intensity at high q with varying copolymer concentration are not very pronounced. This justifies the omission of the unimer scattering contribution in the 


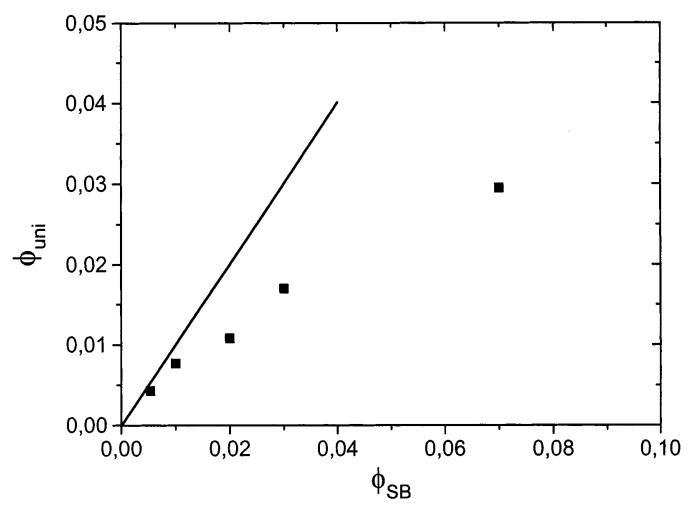

Figure 4. Dependence of the unimer content $\phi_{\text {uni }}$ on the overall block copolymer volume fraction $\phi_{\mathrm{SB}}$.

data analysis of core contrast measurements. However, also in core contrast a small effect of the unimer scattering on the signal intensities at high q is visible. As can be seen in Figure 3a, the slope at high q varies with concentration. At high concentrations $(7 \%, \nabla)$ the final slope is almost $(-4)$ as expected for a spherical form factor. With decreasing concentration and increasing unimer part, the slope more and more adopts the value of -2 , which is expected for single chain scattering. The smaller slope of the $0.5 \mathrm{wt} \%$ sample may be explained by imperfect corrections resulting from the large incoherent background of the protonated matrix polymer and will not be discussed further. Such an influence on the slope cannot be observed in shell contrast measurements because the single chain scattering also dominates the scattering of the micelle corona. In Figure $3 b$ one finds a constant sl ope of -2 at high q for all concentrations.

The variation of the unimer concentration $\phi_{\text {uni }}$ with the overall block copolymer content $\phi_{\mathrm{SB}}$ is shown in Figure 4.

For a system with a well-defined $\mathrm{cmc}$, for example low molecular weight surfactants in water, at concentrations $\phi<\mathrm{cmc}$ all molecules are found as unimers $\left(\phi_{\text {uni }}=\phi\right)$. If the concentration in this case exceeds the $\mathrm{cmc}$, additional molecules are built into micelles whereas $\phi_{\text {uni }}$ remains constant $\left(\phi_{\text {uni }}=\mathrm{cmc}\right)$. The line in Figure 4 corresponds to $\phi_{\mathrm{uni}}=\phi_{\mathrm{SB}}$. It is obvious that the PS-PB block copolymer/PB homopolymer blend does not behave like surfactant solutions since a sharp cmc could not be observed. Although micelles are al ready formed in the $0.5 \mathrm{wt} \%$ blend, beyond that point the unimer concentration is still increasing. A similar behavior could be observed for amphiphilic bl ock copolymers in solution. ${ }^{20}$ Here, a similar nonlinear increase in the unimer concentration was found in a concentration range of $0.5-2 \%$, which finally ended in a plateau value. Such a plateau is not yet reached in Figure 4 although the values are slowly leveling off. We therefore conclude that in a block copolymer/homopolymer melt the cmc is less well-defined than in a low molecular selective solvent. 20

Effective Size of Swollen Micelles. Importantly, our investigations by SANS also revealed information about the degree of swelling of the micelle by the homopolymer as well as the corona density profile itself. The swelling of the micelles and the effective particle size could be determined from the overall micellar radius $R_{M}$, but also from the fit parameters of the structure factor $\mathrm{R}_{\mathrm{HS}}$ and $\mathrm{V}_{\mathrm{HS}}$.

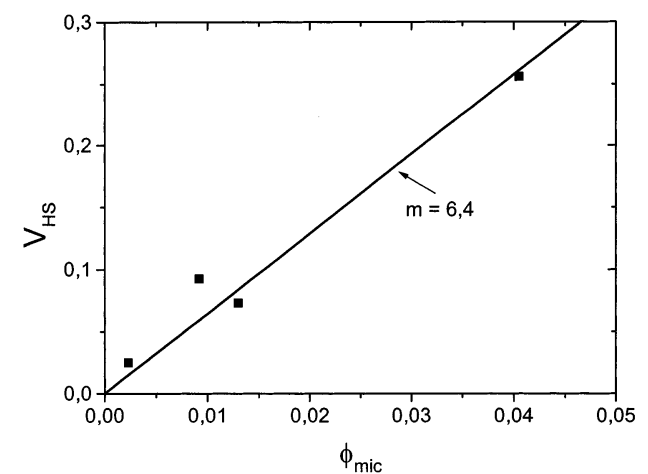

Figure 5. Hard-sphere volume fraction $\mathrm{V}_{\mathrm{HS}}$, determined from the structure factor, in dependence of the volume fraction of aggregated block copolymer $\phi_{\text {mic. }}$.

Figure 5 shows the dependence of the effective hardsphere volume fraction $V_{\mathrm{HS}}$ on the volume fraction $\phi_{\text {mic }}$ of aggregated block copolymer (corrected for the unimer part!). The direct proportional ity of $\mathrm{V}_{\mathrm{HS}}$ and $\phi_{\text {mic }}$ underlines the validity of our analysis of the actual micelle concentration and the corresponding unimer concentration. The slope of $m=6.4$, which should be equal to 1 for nonswollen micelles, shows that the homopolymer penetrates into the corona and thereby increases the effective particle size. This is also reflected by the effective hard-sphere radius, which is much larger than the value expected for nonswollen dry micelles of $\mathrm{R}=$ $200 \AA$ calculated using eq 6

$$
R_{\text {ges }}=R_{\text {Kern }} \sqrt[3]{1+\frac{M_{P B}}{M_{P S}} \frac{\rho_{P S}}{\rho_{P B}}}
$$

with $M_{P I}$ and $M_{P S}$ being the molecular masses of the PS and PB block and $\rho_{\mathrm{PS}}$ and $\rho_{\mathrm{PB}}$ the respective polymer densities. I mportantly, the values for $\mathrm{R}_{\mathrm{HS}}$ (Table 3) show no systematic dependence on concentration. The scattering in the numbers is caused by the difficulty in the structure factor analysis at low concentrations. If the mean value $\left\langle\mathrm{R}_{\mathrm{HS}}\right\rangle=370 \AA$ is compared to the radius of the nonswollen micelle, the effective micelle radius is enlarged by a factor of 1.8 , which nicely corresponds to the volume swelling of $6.4 \approx 1.8^{3}$ determined from Figure 5. Interestingly, the constant effective particle radius (Table 3 ) and the proportionality of hard-sphere volume fraction and copolymer micelle concentration (Figure 5) show that in the concentration regime investigated here $\left(\phi_{\text {mic }}<0.05\right)$ a decrease in particle size swelling with concentration has not yet been found.

Also, the micelle radius $\mathrm{R}_{\mathrm{M}}$ determined from the form factor in shell contrast is constant over the studied concentration range. But in comparison to $R_{H S}$ (about $370 \AA$ ), it is significantly smaller (about $240 \AA$ ). $R_{M}$ is the size of a spherical micelle as determined by the extension of the micellar corona. Since our copolymer micelles are neither polar nor charged, they should only interact at particle contact. Therefore, the large difference in particle size $R_{M}$ and effective hard-sphere radius $\mathrm{R}_{\mathrm{HS}}$, the latter providing a direct measure for the range of interparticle interactions, is difficult to understand. A possible explanation might be the existence of steric repulsion between the micelles, which is mediated by a few dangling corona ends. Whereas the majority of the corona polymer is packed densely around the micellar core and thereby defines the micellar size $R_{M}$ found in 


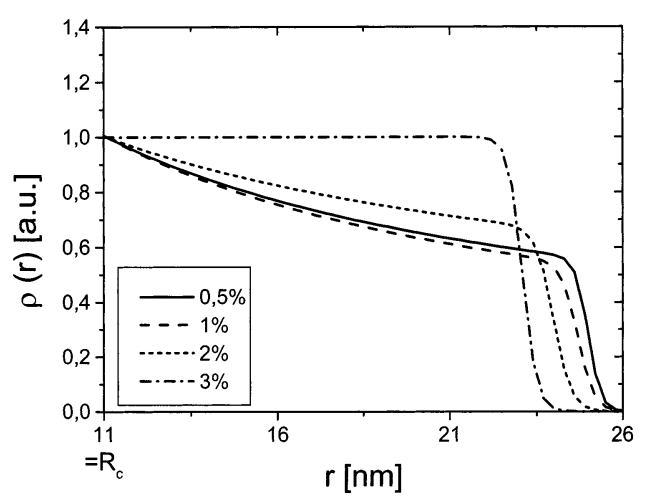

Figure 6. Density profiles for the different blends obtained from the fits. The relative densities have been rescaled to the same density at $r=R_{c}$ for all blends.

shell contrast, a few corona chains extend far beyond $\mathrm{R}_{\mathrm{M}}$ into the homopolymer matrix and thereby cause an increase in interaction range compared to $\mathrm{R}_{\mathrm{M}}$. To check these assumptions, we will consider the density profile of the micelle corona in more detail in the next section.

Density Profile of the Corona of Swollen Micelles. Figure 6 shows the density profiles obtained from the fits on the scattering intensities in shell contrast at various copolymer concentrations.

For lower concentrations, a slowly decreasing density profile is detected. This profile, however, does not continuously decay toward zero as expected from the Daoud and Cotton model for star polymers ${ }^{11}$ but still exhibits a significant segment density close to the cutoff radius $\mathrm{R}_{M}=250 \AA$. F or the highest analyzed concentration ( 3 wt \%) such a decay is not detected anymore, but the segment density is constant up to a cutoff radius $\mathrm{R}_{\mathrm{M}}=230 \AA$. This implies that the decrease of the density profile at lower block copolymer content is merely an effect of the $\mathrm{cmc}$, found at very low concentrations where the micellar structure is not yet welldefined. The constant segment density profile (eq $1, x$ $=0$ ) of the copolymer corona at 3 wt \% is in contradiction to the scenario expected from the block copolymer composition. The fact that the corona block is much longer than the core block implies rather a starlike structure for the resulting micelles, which should give an exponent of $x=4 / 3$. This picture holds for micelles in solution, ${ }^{12}$ but our results show that this is not the case in the melt, where we find a constant corona density. Our finding nicely confirms the scenario described in the preceding section: the effective particle size of swollen micelles in a homopolymer matrix is mainly determined by a few dangling ends, which cause steric repulsion but, due to their low segment density, do not contribute to the mi celle radius detected in shell contrast. The latter, on the other hand, is defined by a slightly swollen corona shell of homogeneous segment density. In this respect, the decrease of swelling with increasing micelle concentration found in our previous dynamical studies ${ }^{4}$ can be interpreted by a compression and/or interdigitation of the steric stabilization layer, whereas the major part of the corona in the regime of the homogeneous segment density profile basically remains unchanged. In our case, the stabilization layer is on the order of $120 \AA$, in comparison to a micelle size $\mathrm{R}_{\mathrm{M}}=240 \AA$ and a core size $\mathrm{R}_{\mathrm{PS}}=110 \AA$. This topol ogy is sketched in Figure 7:

Assuming a strongly stretched configuration of the dangling ends, the molecular weight of these should be

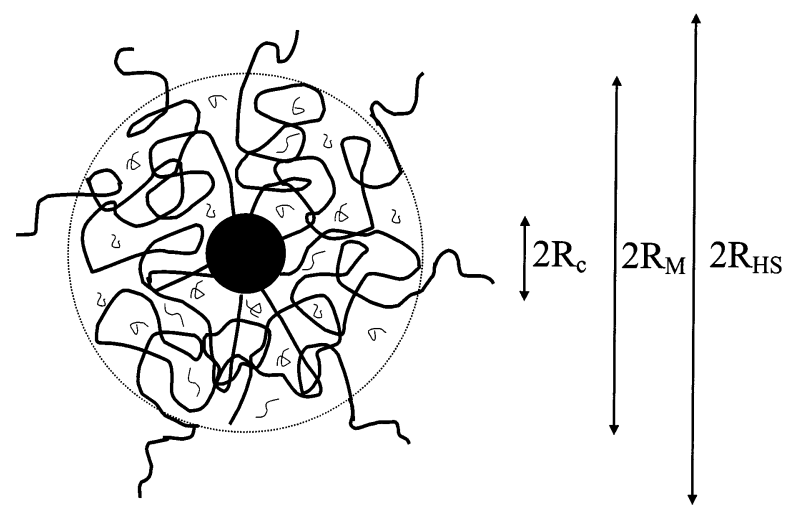

Figure 7. Schematic picture of a block copolymer micelle on the basis of the presented results.

about $5000-10000 \mathrm{~g} / \mathrm{mol}$, which for PB is clearly in the entangl ement regime $\left(M_{e} \sim 2000\right)$. An interesting question in this context is why the strong swelling by the homopolymer chains according to our scattering results does not proceed beyond $R_{M}=240 \AA$. Here, one only might speculate: First, because of the high grafting density close to the PS core of the micelles, corresponding to an average distance of less than $25 \AA$ between neighboring corona arms, the PB homopolymer chains $(\mathrm{M}=8000 \mathrm{~g} / \mathrm{mol}$, unperturbed coil diameter in the melt about $70 \AA^{21}$ ) cannot migrate too far into the micelle for steric reasons. In this respect, the swelling ratio within the region of constant corona segment density $\left(\left(R_{M}-R_{C}\right) /\left(R-R_{C}\right)\right)^{3}=((240-110) /(200-110))^{3}=1.9$ is astounding, since it corresponds to a homopolymer content of about $50 \%$ within this corona regime of the micelle. On the other hand, the corona arms of an individual micelle could be expected to be highly interentangled, which, besides the steric effects, may further limit the maximum swelling of this inner corona part.

The scenario depicted in Figure 7 has important consequences on the solid-liquid transition of swollen spherical micelles in a homopolymer matrix. If the decrease in particle swelling with increasing concentration is mainly caused by a structural change in the steric stabilization layer of the dangling corona ends, the swelling at the solid liquid transition should be given roughly by $R_{M}$ with respect to the radius of the nonswollen micelle. In our case, this leads to an effective volume swelling of $\left(R_{M} / R\right)^{3}=1.7$. In comparison to the hard-sphere glass transition at $\phi=0.64$, our micellar system therefore should freeze at a copolymer concentration of about $0.64 / 1.7=0.37$. From dynamic investigations of PS-PI block copolymer micelles, with block length composition comparable to the PS-PB system studied here, in various matrix homopolymers, we have found a solid-liquid transition at $\phi_{\mathrm{PSPI}}=0.56$ (matrix 1: $M=50000 \mathrm{~g} / \mathrm{mol}$ ) and 0.40 (matrix 2: $M=23000$ $\mathrm{g} / \mathrm{mol}) .{ }^{4} \mathrm{~F}$ or a still shorter matrix homopolymer investigated by Watanabe et al. (matrix 3: $M=5000 \mathrm{~g} / \mathrm{mol})^{2}$ our data analysis yielded a solid-liquid transition at about $\phi_{\mathrm{PSP}}=0.31$. The value $\phi_{\mathrm{PSPB}}=0.37$ estimated from simple considerations as sketched above for the matrix homopolymer $\mathrm{M}=8000 \mathrm{~g} / \mathrm{mol}$ investigated here nicely fits in this series. This seems to confirm the assumption that swollen copolymer micelles in a homopolymer matrix consist of basically three regions: (1) segregated core, (2) comparatively slightly swollen inner corona layer with homogeneous density profile of block segments, and (3) an outer layer of highly stretched corona chains with very low segment density that 
determines the effective particle size by steric stabilization. The decrease in effective particle size with increasing micelle concentration is caused mainly by changes in the structure of this outer layer (compression/interdigitation).

Finally, we would like to mention that we tried to investigate the corona density profile also for copolymer micelles embedded in a much longer homopolymer matrix. However, scattering signals obtained from PSPB micelles embedded in a PB matrix of $\mathrm{M}=100000$ $\mathrm{g} / \mathrm{mol}$, that is about twice as long as the corona block, indicated demixing of micelles and matrix. This demixing caused by depletion interactions ${ }^{22,23}$ is expected in case the matrix chains are longer than the corona hairs, as has been shown by previous experimental investigations of a similar system of hairy nanospheres in a homopolymer melt. ${ }^{24}$ Since the structure factor could not easily be determined for these demixed samples, it was al so not possible to analyze the particle form factor and therefore the corona density profile of the micelles. However, according to our dynamic studies, ${ }^{4}$ we expect the corona swelling in region 2 (Figure 7) to systematically decrease with increasing length of the matrix chains. Also, the stretching of the corona ends (region 3 , Figure 7) should decrease accordingly.

\section{Conclusions}

Neutron scattering experiments on block copolymer/ homopolymer blends with various block copolymer concentrations revealed the existence of a very broad cmc. The decreasing normalized scattering intensity with decreasing concentration lead to the assumption that the amount of free block copolymer chains in the samples could not be ignored. The analysis of the data with respect to unimers showed that in contrast to surfactants, which show a sharp cmc, and block copolymer micelles in a selective solvent, where a similar but narrower transition zone is found, the unimer concentration in the blends has not reached a constant value at a block copolymer content up to 7 wt \% (Figure 4).

From fits of the form and structure factor in core and shell contrast, where the unimer content was taken into account, the radii of the core and the whole micelle as well as the structure parameters for a hard-sphere model were obtained. In the investigated concentration range, the size of the micelles remained constant within the error of the analysis. A strong swelling of the micelle corona by the short homopolymer matrix was seen as expected: the dry nonswollen micelle of radius $R=20$ $\mathrm{nm}$ is swollen to a size of $\mathrm{R}_{\mathrm{M}} \approx 24 \mathrm{~nm}$, determined from the shell contrast form factor. From the structure factor analysis an even larger effective radius of $\mathrm{R}_{\mathrm{HS}} \approx 37 \mathrm{~nm}$ was found. This large increase in effective radius is in our opinion caused by a steric repulsion of the micelles mediated by dangling chain ends which reach out into the melt far beyond the corona dimensions $\mathrm{R}_{\mathrm{M}}$.

To support these assumptions, the corona segment density profile was determined using a variable density profile for the outer shell of the micelle. We found that the corona has a constant segment density. Only the micelles in lower concentrated blends showed a small decrease in density to a minimum of $50 \%$ of the density found directly at the core. This is considered as a $\mathrm{cmc}$ effect. Our results show that the micelles in the melt are rather compact particles although from the block length ratio, in analogy to previous studies of block copolymer micelles in solution, a more starlike behavior would have been expected. According to our results, a swollen copolymer micelle in a homopolymer matrix basically consists of three regions: the polystyrene forms a spherical core of radius $R_{c}$, surrounded by $a$ corona of the PB block which is homogeneously swollen by homopolymer matrix chains. This corona has a constant segment density with respect to the block copolymer segments, which reaches out to the radius $\mathrm{R}_{\mathrm{M}}$. Beyond this radius there are dangling chain ends reaching out from the corona, which cause a repulsive micellar interaction that leads to an effective radius $R_{H S}$ relevant for the structure formation and the dynamics in the blend. The decrease in swelling of the particle size with increasing micelle concentration found in previous dynamic investigations seems to be caused by structural changes in this outer corona region only, whereas the regime with constant segment density seems to remain unchanged. Detailed investigations by SANS at very high micelle concentrations should reveal the validity of these assumptions: well above the $\mathrm{cmc}$, the corona segment density profile is expected to remain basically unchanged with increasing concentration, whereas the structure factor should indicate a strong decrease in effective particle size and, corrrespondingly, in particle swelling.

Acknowledgment. Financial support by the Deutsche F orschungsgemeinschaft, Grants DF G Scha620/2$1,-2$, is gratefully acknowledged. Also, we thank D. Schwahn for his help with SANS measurements on test samples and H. Kaya for his support in the model fitting.

\section{References and Notes}

(1) Watanabe, H. Acta Polym. 1997, 48, 215.

(2) Watanabe, H.; Sato, T.; Osaki, K.; Hamersky, M. W.; Chapman, B. R.; Lodge, T. P. Macromolecules 1998, 31, 3740.

(3) Gohr, K.; Pakula, T.; Tsutsumi, K.; Schärtl, W. Macromolecules 1999, 32, 7156.

(4) Gohr, K.; Schärtl, W. Macromolecules 2000, 33, 2129.

(5) Martin, I. J .; Wang, Z.-G. J . Phys. Chem. 1995, 99, 2834.

(6) Shull, K. R. Macromolecules 1996, 29, 2659.

(7) Berney, C. V.; Cheng, P.-L.; Cohen, R. E. Macromolecules 1988, 21, 2235.

(8) Grest, G. S. J . Chem. Phys. 1997, 105, 5532.

(9) Birshtein, T. M.; Zhulina, E. B. Polymer 1989, 30, 170.

(10) Halperin, A. Macromolecules 1987, 20, 2943.

(11) Daoud, M.; Cotton, J . P. J . Phys. (Paris) 1982, 43, 532.

(12) Willner, L.; Poppe, A.; Allgaier, J .; Monkenbusch, M.; Lindner, P.; Richter, D. Europhys. Lett. 2000, 51, 628.

(13) Morton, M.; Fetters, L. J . Rubber Chem. Technol. 1975, 48, 359.

(14) Hadjichristidis, N.; Iatrou, H.; Pispas, S.; Pitsikalis, M. J Polym. Sci., Part A: Polym. Chem. 2000, 38, 3211.

(15) Aminabhavi, T. M. J . Macromol. Sci., Chem. 1983, 19, 687.

(16) Fetters, L. J .; Hadjichristidis, N.; Lindner, J . S.; Mays, J . W. J. Phys. Chem. Ref. Data 1994, 23, 619.

(17) Dozier, W. D.; Huang, J . S.; Fetters, J . L. Macromolecules 1991, 24, 2810.

(18) Pedersen et al. J . Appl. Crystallogr. 1990, 23, 321.

(19) Higgins, J. S.; Benoit, H. C. Polymers and Neutron Sattering; Clarendon Press: Oxford, 1994

(20) Kreitschmann, M. Dissertation, Aachen, 1998.

(21) Polymer Handbook; Barndrup, J ., I mmergut, E. H., Eds.; Wiley \& Sons: New York, 1989.

(22) Vrij, A. Pure Appl. Chem. 1976, 48, 471.

(23) Asakura, S.; Oosawa, F. J . Chem. Phys. 1954, 22, 1255.

(24) Lindenblatt, G.; Schärtl, W.; Pakula, T.; Schmidt, M. Macromolecules 2000, 33, 9340 .

MA020881P 